\title{
Custo benefício dos nutrientes dos alimentos consumidos no Brasil
}

\author{
Cost-benefit ratio of the nutrients of the food consumed in Brazil
}

Kennya Beatriz Siqueira (https://orcid.org/0000-0001-6727-7774) ${ }^{1}$

Mirella Lima Binoti (https://orcid.org/0000-0001-5274-8588) ${ }^{2}$

Renato Moreira Nunes (https://orcid.org/0000-0003-4397-5898) ${ }^{2}$

Cristiano Amancio Vieira Borges (https://orcid.org/0000-0001-8120-3288) ${ }^{1}$

Amanda Fernandes Pilati (https://orcid.org/0000-0002-7431-4024) ${ }^{1}$

Guilherme William Marcelino (https://orcid.org/0000-0002-0167-2242) ${ }^{3}$

Marco Antônio Sundfeld da Gama (https://orcid.org/0000-0002-1970-5882) ${ }^{1}$

Paulo Henrique Fonseca da Silva (https://orcid.org/0000-0002-4965-8714) ${ }^{2}$
${ }^{1}$ Embrapa Gado de Leite. R. Eugênio do Nascimento 610 Dom Bosco. 36038-330 Juiz de Fora MG Brasil. kennya. siqueira@embrapa.br ${ }^{2}$ Departamento de Nutrição da Universidade Federal de Juiz de Fora. Juiz de Fora MG Brasil.

${ }^{3}$ Departamento de

Estatística da Universidade Federal de Juiz de Fora. Juiz de Fora MG Brasil.

\begin{abstract}
Nutrient deficiency is one of the major problems of the human population today. Studies indicate that consumers consider that the price of food is more relevant than its nutritional value. Therefore, this work sought to compare the costs of nutrients provided by typical foods found in the Brazilian diet. Food prices available in virtual markets were collected, and the costs to meet $30 \%$ of the daily recommendations for a healthy adult were calculated for eight nutrients. Poultry was found to be the cheapest source of protein. Cereal fiber had the lowest cost for both fiber and iron. The cheapest sources of calcium were Ovaltine and dairy products, whereas cow's liver was the best ranked food for vitamin A. Vitamin $C$ was found to be the cheapest nutrient in the Brazilian diet, with acerola juice being the cheapest source of this nutrient. Dairy products occupied the first six positions in the ranking of vitamin $D$, while nuts and seeds were the cheapest sources of vitamin E. The results showed that Brazilian consumers can meet the recommended intake of critical nutrients at a low cost through the purchase of the best ranked foods. However, this ranking should be weighted according to the food quality, as recommended in the New Dietary Guidelines for the Brazilian Population.

Key words Nutrition, Public health, Nutrient deficiency, Costs
\end{abstract}

Resumo Um dos maiores problemas atuais da população é a deficiência de nutrientes. Estudos indicam que consumidores consideram o preço dos alimentos mais relevante do que os valores nutricionais. Assim, este trabalho objetivou comparar o custo dos nutrientes de alimentos típicos da dieta brasileira, realizando coletas de preços em mercados virtuais e calculando o custo para se atingir 30\% das necessidades diárias de um adulto saudável para oito nutrientes. A carne de frango foi a fonte mais barata de proteina. A fibra de cereal teve o menor custo para fibra e ferro. As fontes mais baratas de cálcio foram o Ovomaltine e os lácteos, e o fígado bovino foi o alimento melhor ranqueado para vitamina $A$. A vitamina $C$ foi o nutriente mais barato na dieta brasileira, e o suco de acerola a fonte mais barata deste nutriente. Os lácteos ocuparam as seis primeiras posições no "ranking" da vitamina $D$, e nozes e sementes apresentaram o menor custo para vitamina E. Os resultados mostraram que os consumidores brasileiros podem alcançar a ingestão recomendada de nutrientes considerados críticos a um baixo custo por meio da aquisição dos alimentos melhor ranqueados. No entanto, esse ranqueamento deve ser ponderado em função da qualidade do alimento, conforme preconiza o Novo Guia Alimentar para a População Brasileira.

Palavras-chave Nutrição, Saúde pública, Deficiência nutricional, Custos 


\section{Introdução}

Um dos maiores problemas da população mundial na atualidade é a deficiência de nutrientes. Conhecida como fome oculta (hidden hunger), a deficiência de nutrientes atinge mais de 2 bilhões de pessoas ou uma em cada três no mundo, superando, assim, tanto o número de pessoas que sofre de fome crônica ou desnutrição (795 milhões), quanto o de pessoas com sobrepeso ( 1,5 bilhões) ${ }^{1}$.

No Brasil, dados da Pesquisa de Orçamentos Familiares (POF) 2008-2009 mostram que a maior parte da população apresenta deficiência de pelo menos um nutriente, sendo os principais as vitaminas $\mathrm{E}$ e $\mathrm{D}$, que atingem mais de $90 \%$ dessa população ${ }^{2}$. Também são encontradas elevadas prevalências de inadequação para magnésio e cálcio ${ }^{2}$.

Ignorada até recentemente, a deficiência nutricional de vitaminas e minerais, que não apresenta sintomas clínicos e que era previamente considerada de menor relevância, prejudica o desenvolvimento intelectual, cognitivo e físico, causa doenças e mortes prematuras, e condena um terço do mundo a viver em condições físicas e mentais abaixo do nível ótimo ${ }^{3}$. Com isso, o custo da fome oculta para a sociedade é elevado, pois envolve gastos com saúde, previdência, perda de produtividade laboral, mortes prematuras, etc. Portanto, o grande desafio do mundo moderno não é mais alimentar a população mundial, mas sim nutrir essa população.

As razões para a deficiência de nutrientes podem ser várias: custo dos produtos alimentícios, disponibilidade dos alimentos, tradição, falta de conhecimento nutricional, preferências sensoriais, entre outras. No entanto, estudos têm mostrado que os consumidores consideram as questões nutricionais menos relevantes do que sabor e preço dos alimentos ${ }^{4-7}$. Nos Estados Unidos, Darmon et al. ${ }^{8}$ e Monsivais e Drewnowski ${ }^{9}$ identificaram que a aquisição de alimentos é mais influenciada pelo preço do que pelo valor nutricional. Resultado semelhante foi encontrado por Drewnowski et al..$^{10}$ na França e Ward et al..$^{11}$ na Austrália, indicando que o preço dos alimentos pode afetar a qualidade da dieta da população. $\mathrm{O}$ aumento do preço dos alimentos básicos previsto pela FAO-OECD ${ }^{12}$ para a próxima década pode, portanto, restringir a dieta das pessoas e gerar grandes impactos socioeconômicos e barreiras para o desenvolvimento sustentável dos países.

Diante do exposto, pode-se assumir que o Brasil pode encontrar dificuldades econômicas, sociais e do âmbito da saúde devido à má alimentação da população. Nesse sentido, torna-se oportuno associar o conceito de saúde e nutrição com o valor pago pelos alimentos, o que foi feito neste trabalho por meio da comparação do custo dos nutrientes de alimentos típicos da dieta brasileira, visando identificar produtos que apresentem vantagem em termos da relação custo benefício dos nutrientes.

\section{Metodologia}

O presente estudo teve como referência a metodologia proposta por Wenhold e Leighton ${ }^{13}$ para avaliar o custo dos nutrientes dos alimentos consumidos no Brasil em termos do atendimento de $30 \%$ das recomendações nutricionais diárias ou RDAs (Recommended Dietary Allowances) para adultos saudáveis. Esse percentual nutricional proposto por Wenhold e Leighton ${ }^{13}$ se justifica pelo fato de que nenhum alimento, por si só, consegue atender todas as necessidades diárias de um indivíduo.

Para esta análise, foi considerada a lista de alimentos mais consumidos no Brasil, segundo a Pesquisa de Orçamentos Familiares (POF) 2008$2009^{2}$, que engloba produtos típicos e tradicionais, bem como alguns alimentos processados e de marca. A mesma pesquisa foi empregada para coleta dos atributos nutricionais dos alimentos selecionados. Com a tabela de composição nutricional, cada alimento foi convertido em nutrientes. Segundo o $\mathrm{FDA}^{14}$, são considerados alimentos saudáveis aqueles que contêm os seguintes nutrientes: cálcio, proteína, vitamina A, fibras, vitamina $\mathrm{C}$ e ferro. Portanto, esses nutrientes foram incluídos na análise. Adicionalmente, foram incluídas as vitaminas $\mathrm{D}$ e $\mathrm{E}$, por serem os nutrientes que apresentam maiores índices de prevalência de inadequação na população brasileira ${ }^{2}$.

Devido às dimensões continentais do Brasil, os preços dos alimentos foram cotados via internet, reduzindo, assim, os gastos elevados de uma coleta in loco em todo o território nacional. Foi realizado um levantamento dos supermercados mais representativos em cada estado da federação. No entanto, nas Regiões Norte e Nordeste não foi encontrado nenhum mercado representativo. Ao todo, foram realizadas coletas de preços em 15 supermercados distribuídos em 9 estados.

Para cada produto, foi coletado o menor preço no varejo (independente da marca), sendo este preço, posteriormente, convertido para $100 \mathrm{~g}$ do 
produto. Preços promocionais não foram considerados.

Para evitar os efeitos de inflação e de sazonalidade, a coleta de preços ocorreu duas vezes no ano de 2016 (nos meses de abril e outubro) de forma concomitante, ou seja, dentro do mesmo mês em todos os locais. Na sequência, foram calculados os preços médios para o Brasil.

Com as informações anteriores, foi possível calcular o custo dos nutrientes dos alimentos usando a seguinte fórmula:

$$
P_{n p}=\left(N_{n} \cdot P_{p}\right) / Q_{n}
$$

em que: $P_{n p}=$ Custo do nutriente $n$ no alimento $p$ (expresso em R $\$$ ); $N_{n}=$ Quantia equivalente a $30 \%$ da recomendação nutricional diária do nutriente $n$ (expressa em g, $\mathrm{d}$ ou mcg); $P_{p}=$ Preço de 100 gramas do alimento $p(\mathrm{R} \$) ; Q_{n}^{p}=$ Quantidade do nutriente $n$ em 100 gramas do alimento ( $\mathrm{g}$, d ou $\mathrm{mcg}$ ). [conferir cuidadosamente trechos em vermelho]

A comparação do resultado deste cálculo para cada nutriente de cada produto permitiu identificar os alimentos mais baratos e nutritivos em termos de cada nutriente de interesse.

\section{Resultados}

Do total de alimentos presentes na POF 2008$2009^{2}$, apenas 443 foram considerados na análise devido à disponibilidade desses produtos nos supermercados consultados. Os alimentos foram agrupados em 13 categorias de produtos: verduras, hortaliças e derivados; frutas e derivados; pescados e frutos do mar; leguminosas e derivados; leite e derivados; ovos e derivados; carnes e derivados; bebidas; cereais e derivados; nozes e sementes; produtos açucarados, diet e light e outros alimentos. Não foram incluídas bebidas alcoólicas, óleos e gorduras.

As Tabelas 1 e 2 apresentam os principais alimentos no "ranking" do custo dos 8 nutrientes avaliados na pesquisa.

Os resultados da pesquisa indicam que o frango e seus derivados são as fontes mais baratas de proteína no Brasil (Tabela 1). No "ranking" dos alimentos que apresentam menor custo para se atingir 30\% das necessidades diárias de proteína, os 5 primeiros colocados são produtos avícolas. Na sequência, têm-se outros tipos de carnes, nozes e sementes, ovos e produtos lácteos. É interessante notar também que a proteína é o nutriente economicamente mais acessível à população bra- sileira, visto que 144 alimentos e bebidas oferecem 30\% dos requerimentos diários de proteína no Brasil a um custo inferior a $\mathrm{R} \$ 5,00$.

No caso das fibras, o produto que consegue oferecer 30\% das necessidades diárias de um adulto saudável no Brasil a um menor custo é a fibra de cereal $(R \$ 0,35)$, que se destaca por oferecer este nutriente a um valor mais de três vezes menor do que o segundo colocado no "ranking" (coco da Bahia; R\$ 1,19). Do total de produtos analisados, 308 apresentam fibras na sua composição, sendo a salsicha em conserva o alimento que oferece $30 \%$ da RDA de fibra ao maior custo: $\mathrm{R} \$ 2.452,04$.

Além de apresentar o melhor custo benefício para fibras, a fibra de cereal também é o produto melhor ranqueado no custo do ferro: $\mathrm{R} \$ 0,66$. Outra informação interessante sobre o ferro é que o "ranking" desse nutriente é o mais diversificado de todos, apresentando produtos de 9 das 13 categorias analisadas entre os 25 alimentos de melhor custo benefício.

O alimento que oferta $30 \%$ das necessidades nutricionais de cálcio ao menor custo no Brasil é o Ovomaltine. Vale ressaltar que o Ovomaltine é um produto formulado, tendo produtos lácteos (soro de leite em pó e leite em pó integral) como ingredientes que agregam cálcio na formulação. Em acréscimo à importância dos lácteos no aporte de cálcio pelo Ovomaltine, a indicação de preparo do mesmo considera o uso de leite fluido ( 2 colheres ou $20 \mathrm{~g}$ de Ovomaltine em $200 \mathrm{~mL}$ ou um copo de leite fluido). Assim, os lácteos constituem a fonte de cálcio preponderante no Ovomaltine, tanto no produto comercial quanto no produto já preparado para o consumo. Além disso, é importante ressaltar que o Ovomaltine, assim como Neston, Chocomilk, Toddynho, entre outros, são alimentos processados e que sofreram adição de nutrientes na sua formulação. Apesar de terem sido incluídos neste estudo por estarem na lista da POF 2008-2009 como os alimentos mais consumidos do País e por terem sido bem ranqueados para alguns nutrientes, o Novo Guia Alimentar para a População Brasileira recomenda o consumo de alimentos na sua forma natural, sem a adição de ingredientes.

Os seis alimentos que se seguem no "ranking" do cálcio são produtos lácteos: leite UHT integral, leite pasteurizado, leite semidesnatado, leite em pó integral, leite em pó desnatado e leite UHT desnatado. Além disso, dos 25 produtos que apresentaram melhor custo benefício para o cálcio no Brasil, 15 são derivados do leite. De todos os lácteos avaliados (43), apenas 6 apresentaram um custo superior a $\mathrm{R} \$ 10,00$ para o cálcio. 
Tabela 1. Rankings dos custos de proteína, fibra, cálcio e ferro dos alimentos consumidos no Brasil (em R\$/30\% da RDA).

\begin{tabular}{|c|c|c|c|c|c|c|c|c|}
\hline Posição & Produto & Proteína & Produto & Fibra & Produto & $\mathrm{Ca}$ & Produto & $\mathrm{Fe}$ \\
\hline 1 & Frango inteiro & 0,59 & Fibra de cereal & 0,35 & Ovomaltine & 0,87 & Fibra de cereal & 0,66 \\
\hline 2 & Frango em pedaços & 0,68 & Coco da Bahia & 1,19 & Leite integral & 0,97 & Marisco & 0,93 \\
\hline 3 & Peito de galinha & 0,72 & Farinha de milho & 1,20 & Leite pasteurizado & 1,00 & Coentro & 0,93 \\
\hline 4 & Steak de frango & 0,78 & $\begin{array}{l}\text { Farinha de } \\
\text { mandioca }\end{array}$ & 1,30 & Leite semidesnatado & 1,04 & Neston & 1,23 \\
\hline 5 & Filé de frango & 0,82 & Farofa pronta & 1,51 & Leite em pó integral & 1,09 & Fígado bovino & 1,27 \\
\hline 6 & Toucinho & 0,83 & $\begin{array}{l}\text { Semente de } \\
\text { linhaça }\end{array}$ & 1,54 & $\begin{array}{l}\text { Leite em pó } \\
\text { desnatado }\end{array}$ & 1,11 & Milk shake diet & 1,35 \\
\hline 7 & Amendoim moído & 0,86 & Milho (em grão) & 1,64 & Leite desnatado & 1,16 & Farinha de milho & 1,36 \\
\hline 8 & Asa de galinha & 0,87 & Coco & 1,68 & Creme de milho & 1,17 & Diet shake & 1,55 \\
\hline 9 & Fígado bovino & 0,89 & $\begin{array}{l}\text { Amendoim } \\
\text { moído }\end{array}$ & 1,77 & Leite condensado & 1,25 & $\begin{array}{l}\text { Amendoim } \\
\text { moído }\end{array}$ & 1,56 \\
\hline 10 & Ovo de codorna & 0,89 & Farinha de aveia & 1,83 & Achocolatado em pó & 1,50 & Pão de forma & 1,60 \\
\hline 11 & Costela suína & 1,18 & Aveia em flocos & 1,85 & $\begin{array}{l}\text { Achocolatado em } \\
\text { pó light }\end{array}$ & 1,52 & Ovomaltine & 1,70 \\
\hline 12 & $\begin{array}{l}\text { Amendoim } \\
\text { (em grão) }\end{array}$ & 1,18 & Pão light & 1,89 & Leite de soja em pó & 1,90 & Soja em grão & 1,73 \\
\hline 13 & $\begin{array}{l}\text { Hambúrguer de } \\
\text { carne }\end{array}$ & 1,19 & Laranja & 1,92 & Queijo colonial & 2,00 & Rapadura & 1,74 \\
\hline 14 & Tatu & 1,32 & Banana & 1,93 & Neston & 2,02 & Yakissoba & 1,87 \\
\hline 15 & Fiambre & 1,32 & Feijão de corda & 2,03 & Leite de cabra & 2,09 & Farinha de aveia & 2,02 \\
\hline 16 & Farinha de milho & 1,35 & Germe de trigo & 2,05 & Mumu & 2,10 & Germe de trigo & 2,04 \\
\hline 17 & Bife rolê cru & 1,36 & Abacate & 2,06 & Mozzarella & 2,12 & Aveia em flocos & 2,05 \\
\hline 18 & Fibra de cereal & 1,40 & Pão integral light & 2,07 & Coentro & 2,12 & Coco da Bahia & 2,09 \\
\hline 19 & Carne moída & 1,41 & Chuchu & 2,09 & Queijo prato & 2,13 & $\begin{array}{l}\text { Amendoim (em } \\
\text { grão) }\end{array}$ & 2,14 \\
\hline 20 & Apresuntado & 1,47 & Maracujá & 2,11 & Chocomilk & 2,18 & Espinafre & 2,17 \\
\hline 21 & Bacon & 1,48 & Arroz integral & 2,15 & Iogurte desnatado & 2,18 & Feijão & 2,25 \\
\hline 22 & Linguiça de frango & 1,52 & Pipoca light & 2,18 & Queijo ralado & 2,27 & Bisnaguinha & 2,47 \\
\hline 23 & Ovo de galinha & 1,58 & Goiaba & 2,26 & Queijo minas & 2,30 & $\begin{array}{l}\text { Biscoito salgado } \\
\text { light }\end{array}$ & 2,89 \\
\hline 24 & Leite integral & 1,59 & Limão & 2,29 & Queijo provolone & 2,32 & Molho de tomate & 2,91 \\
\hline 25 & Rabada de boi & 1,60 & $\begin{array}{l}\text { Biscoito salgado } \\
\text { integral }\end{array}$ & 2,34 & Mozzarella light & 2,35 & Orégano & 2,91 \\
\hline
\end{tabular}

Fonte: Resultados da Pesquisa.

Para satisfazer 30\% das recomendações diárias de vitamina $\mathrm{A}$ ao menor custo no Brasil, a população deve consumir fígado bovino. No entanto, muitas verduras, legumes e produtos lácteos também oferecem este nutriente a um baixo custo.

Já a vitamina $\mathrm{C}$ é o nutriente mais barato no Brasil dentre os avaliados no presente estudo. Com apenas R 0,01 é possível suprir 30\% das necessidades diárias desse nutriente consumindo suco de acerola. Além disso, 21 alimentos e bebidas oferecem $30 \%$ das RDAs de vitamina $\mathrm{C}$ a menos de R\$1,00. Sucos, frutas e verduras, além de doces de frutas, figuram nas primeiras colocações no "ranking" do custo de vitamina C no Brasil.

Assim como evidenciado para fibras, uma quantidade pequena de produtos alimentícios apresenta vitamina D na sua composição. Dos 443 alimentos analisados, apenas 172 possuíam vitamina D. Desse total, os derivados lácteos se destacaram: os seis primeiros colocados nesse "ranking" foram produtos da indústria de laticínios e 11 dos 25 produtos com melhor custo benefício para a vitamina $\mathrm{D}$ também eram lácteos.

Para vitamina E, o melhor custo benefício no Brasil está nas nozes e sementes, que ocuparam as 
Tabela 2. Rankings dos custos das vitaminas A, C, D e E dos alimentos consumidos no Brasil (em R $\$ / 30 \%$ da RDA).

\begin{tabular}{|c|c|c|c|c|c|c|c|}
\hline Produto & $\begin{array}{c}\text { Vit } \\
\text { A }\end{array}$ & Produto & $\begin{array}{c}\text { Vit } \\
\text { C }\end{array}$ & Produto & $\begin{array}{l}\text { Vit } \\
\text { D }\end{array}$ & Produto & $\begin{array}{c}\text { Vit } \\
\text { E }\end{array}$ \\
\hline Fígado bovino & 0,04 & Suco acerola & 0,01 & Leite semidesnatado & 1,19 & Germe de trigo & 0,49 \\
\hline Patê de fígado & 0,11 & Goiaba & 0,13 & Leite integral & 1,38 & Amendoim moído & 0,71 \\
\hline Inhame & 0,18 & Laranja & 0,20 & Leite pasteurizado & 1,41 & Amendoim (em grão) & 0,98 \\
\hline Cenoura & 0,20 & Coentro & 0,35 & Leite em pó integral & 1,54 & Milk shake diet & 1,06 \\
\hline Batata doce & 0,22 & Lima & 0,41 & Leite em pó desnatado & 1,55 & Torrone & 1,13 \\
\hline Margarina & 0,33 & Suco laranja & 0,42 & Leite desnatado & 1,76 & Diet shake & 1,22 \\
\hline Margarina light & 0,33 & Pimentão & 0,43 & Sardinha em conserva & 1,99 & Neston & 1,40 \\
\hline Moranga & 0,39 & Kiwi & 0,45 & Peixe de água doce & 2,29 & Margarina & 1,51 \\
\hline Cenoura amarela & 0,56 & Goiabada & 0,47 & Ovo de codorna & 2,35 & Amendoim apimentado & 1,64 \\
\hline Espinafre & 0,74 & Repolho & 0,50 & Atum em conserva & 2,70 & Abacate & 1,72 \\
\hline Alface & 1,04 & Limão & 0,52 & Neston & 2,80 & Molho de tomate & 1,74 \\
\hline Ovo de codorna & 1,07 & Suco goiaba & 0,62 & Leite de soja em pó & 2,84 & Amêndoa & 1,97 \\
\hline Jardineira (seleta) & 1,13 & Abacaxi & 0,64 & Farinha láctea & 2,96 & Moranga & 2,01 \\
\hline Creme de leite & 1,14 & Mamão & 0,73 & Chocomilk & 3,06 & Pasta de amendoim & 2,10 \\
\hline Couve & 1,20 & Ovomaltine & 0,76 & Creme de milho & 3,57 & Yakissoba & 2,12 \\
\hline Alface orgânica & 1,40 & Tangerina & 0,79 & $\begin{array}{l}\text { Atum em conserva } \\
\text { light }\end{array}$ & 4,13 & Maionese (molho) & 2,19 \\
\hline $\begin{array}{l}\text { Leite em pó } \\
\text { desnatado }\end{array}$ & 1,44 & Melancia & 0,83 & Ovo de galinha & 4,16 & Presuntinho biscoito & 2,53 \\
\hline Ovomaltine & 1,53 & Mariola & 0,84 & Toddynho & 4,35 & Margarina light & 2,88 \\
\hline $\begin{array}{l}\text { Leite } \\
\text { semidesnatado }\end{array}$ & 1,60 & $\begin{array}{l}\text { Suco } \\
\text { maracujá }\end{array}$ & 0,92 & Creme de leite & 4,82 & Ovo de codorna & 2,92 \\
\hline Manteiga & 1,63 & Suco orgânico & 0,92 & Salmão em conserva & 5,35 & Biscoito salgado light & 3,06 \\
\hline Requeijão & 1,75 & Cebolinha & 0,94 & Leite fermentado & 5,46 & Batata doce & 3,09 \\
\hline Leite desnatado & 1,78 & Melão & 1,02 & Vitamina banana & 6,85 & Espinafre & 3,10 \\
\hline Ovo de galinha & 1,89 & $\begin{array}{l}\text { Suco de } \\
\text { goiaba organ. }\end{array}$ & 1,08 & Ovomaltine & 7,01 & Cenoura & 3,28 \\
\hline Milk shake diet & 1,92 & Suco manga & 1,12 & Mortadela & 7,06 & Doce de amendoim diet & 3,52 \\
\hline Requeijão light & 1,98 & Morango & 1,14 & Milk shake diet & 7,19 & Leite de soja em pó & 3,53 \\
\hline
\end{tabular}

Fonte: Resultados da Pesquisa.

três primeiras colocações no ranking, atendendo $30 \%$ das necessidades diárias de um adulto saudável por menos de R\$1,00. Além das nozes e sementes, destacam-se também neste "ranking" muitos produtos industrializados, como por exemplo, margarina, molho de tomate, maionese, etc.

\section{Discussão}

Como atualmente o desafio da maioria dos consumidores está mais relacionado à decisão de compra de alimentos mais saudáveis a um baixo custo do que propriamente ao acesso a quantidades suficientes de alimentos, o presente estudo mostrou que não é necessário investir grande quantidade de recursos para atingir as necessidades nutricionais diárias de nutrientes no Brasil. Existe uma grande variedade de produtos que oferece os oito nutrientes avaliados a baixo custo. No entanto, é preciso levar em conta as fontes alimentares onde esses nutrientes são veiculados. O Guia Alimentar para População Brasileira orienta que alimentos in natura ou minimamente processados, em grande variedade e predominantemente de origem vegetal, sejam a base da dieta ${ }^{15}$.

Com isso, o estudo reforça a recomendação nutricional de um consumo de alimentos diversificados, o que coaduna com os atributos de uma alimentação saudável, que incluem a variedade da dieta. O consumo de vários tipos de alimentos proporciona a ingestão dos diferentes nutrientes, evitando a monotonia alimentar, que limita 
a disponibilidade de nutrientes necessários para atender às demandas fisiológicas e garantir uma alimentação adequada ${ }^{15}$. As variedades dentro de um mesmo grupo de alimentos implicam em maior diversidade no aporte de nutrientes ${ }^{16}$.

De um modo geral, pode-se dizer que os derivados do leite se destacaram na análise apresentando os menores custos para cálcio e vitamina $\mathrm{D}$, e também obtiveram boas colocações nos rankings de proteína e vitamina $\mathrm{A}$, corroborando resultados de pesquisas anteriores. Drewnowski ${ }^{16}$ usou dados da Pesquisa Nacional de Saúde e Nutrição dos EUA e do USDA para calcular o custo dos nutrientes dos lácteos comparados com outros produtos da dieta americana. Os resultados indicaram que o leite e seus derivados são, de longe, as fontes mais acessíveis de cálcio e estão entre as mais baratas de riboflavina, fósforo e vitamina B12. Na África do Sul, Wenhold e Leighton $^{13}$ analisaram a relação custo-benefício de cinco derivados lácteos comparados com vinte comidas típicas da população urbana, a qual envolvia principalmente comida de rua. Os resultados evidenciaram que os derivados lácteos podem suprir as necessidades de cálcio, proteína, vitamina B2 e B12 a um custo razoável.

Uma limitação deste estudo é o fato de considerar apenas um nutriente por vez. Se por um lado isso pode ser útil para orientar a compra de alimentos de pessoas com deficiência em um nutriente específico, como os anêmicos, por exemplo, por outro, essa classificação pode induzir a escolhas inadequadas por não considerar todos os nutrientes do alimento. Isso fica evidente para o Ovomaltine, por exemplo, que se destacou como a fonte alimentar de melhor custo-benefício para cálcio, mas apresenta elevado teor de açúcar e conservantes, os quais podem ser prejudiciais à saúde. Portanto, deve-se ressaltar que a decisão de compra de alimentos deve também considerar outros aspectos, tais como o nível de processamento, adição de ingredientes, etc.
Por associar o valor nutricional ao preço pago pelo produto, os resultados desta pesquisa podem afetar as decisões de compra, pois permitem ao consumidor avaliar melhor o valor pago por cada nutriente dos alimentos adquiridos, especialmente para as classes de baixa renda, que gastam $80 \%$ do seu salário com alimentação. Assim, pessoas com deficiência em um determinado nutriente podem alocar melhor seus recursos financeiros para atender às recomendações nutricionais de forma a melhorar sua saúde e seu bem estar.

Os resultados da pesquisa também contribuem para a elaboração de dietas sustentáveis, conforme recomendado pela $\mathrm{FAO}^{1}$, que são aquelas com baixo impacto para o meio ambiente e que contribuem para a segurança nutricional e alimentar, e para uma vida saudável da presente e da futura geração. Ao otimizar a escolha alimentar dos consumidores, a informação do custo benefício dos nutrientes no Brasil contribui de forma econômica, social e ambiental para o bem-estar da população.

A comparação do custo benefício dos nutrientes dos alimentos consumidos no Brasil pode ajudar na implementação de políticas numa escala global ou regional, de modo a gerar ações de nutrição mais efetivas, auxiliando, assim, o governo brasileiro a atingir os objetivos estabelecidos na Declaração de Roma, que visa à obtenção da segurança alimentar para todos.

Além disso, a exemplo do que vem ocorrendo nos EUA e UE ${ }^{10}$, este tipo de informação pode ser empregada pela indústria na rotulagem dos produtos e também pelos órgãos encarregados de orientar os consumidores e realizar as recomendações alimentares. O presente estudo também vem ao encontro do consenso acerca da necessidade dos sistemas produtivos contribuírem mais efetivamente para melhorar o nível nutricional da população, através de uma abordagem mais ampla e integrada. 


\section{Colaboradores}

KB Siqueira, PHF Silva e MAS da Gama delinearam a pesquisa; KB Siqueira, ML Binoti e RM Nunes escreveram o artigo; AF Pilati e GW Marcelino realizaram a coleta e análise dos dados; PHF Silva, CAV Borges analisou os resultados; KB Siqueira, MAS da Gama e PHF Silva tiveram a responsabilidade principal pelo conteúdo final.

\section{Referências}

1. Food and Agriculture Organization (FAO). Ending malnutrition: from commitment to action. Rome: FAO; 2015.

2. Instituto Brasileiro de Geografia e Estatística (IBGE). Pesquisa de Orçamentos Familiares 2008-2009: tabela de medidas referidas para os alimentos consumidos no Brasil. Rio de Janeiro: IBGE; 2011.

3. Food and Agriculture Organization (FAO). The state of food and agriculture: food systems for better nutrition. Rome: FAO; 2013.

4. Lloyd HM, Paisley CM, Mela DJ. Barriers to the adoption of reduced-fat diets in a UK population. $J A m$ Diet Assoc 1995; 95(3):316-322.

5. Glanz K, Basil M, Maiback E, Goldberg J, Snyder D. Why Americans eat what they do: taste, nutrition, cost, convenience, and weight control concerns as influences on food consumption. J Am Diet Assoc 1998; 98(10):1118-1126.

6. Lennermas M, Fjellstrom C, Becker W, Giachetti I, Schmitt A, Remaut De Winter A, Kearney M. Influences on food choice perceived to be important by nationally-representative samples of adults in the European Union. Eur J Clin Nutr 1997; 51(Supl. 2):S8-S15.

7. Nestel M. The politics of food choice. In: Nestel M, editor. Food Politics: How the Food Industry Influences Nutrition and Health. Los Angeles: University of California Press Ltd.; 2002. p. 358-374.

8. Darmon N, Darmon M, Maillot M, Drewnowski A. A nutrient density standard for vegetables and fruits: nutrients per calorie and nutrients per unit cost. J Am Diet Assoc 2005; 105(12):1881-1887.

9. Monsivais P, Drewnowski A. The Rising Cost of Low-Energy-Density Foods. J Am Diet Assoc 2007; 107(12):2071-2076.

10. Drewnowski A, Monsivais P, Maillot M, Darmon N Low-energy-density diets are associated with higher diet quality and higher diet costs in French adults. $J$ Am Diet Assoc 2007; 107(6):1028-1032.
11. Ward PR, Mamerow L, Henderson J, Taylor AW, Meyer SB, Coveney J. The Social Determinants of Food Purchasing Practices: Who Chooses Price-beforeHealth, Taste-before-Price or Organic Foods in Australia? Food and Nutrition Sciences 2012; 3(4):461-470.

12. Food and Agriculture Organization (FAO), Organization for Economic Co-operation and Development (OECD). [Internet]. 2013 [acessado 2015 Out 5]. Disponível em: http://www.fao.org/index_en.htm.

13. Wenhold F, Leighton C. Dairy: nutritional value for money for South African consumers. IDF Bulletin 461/2013.

14. Food and Drug Administration (FDA). Code of Federal Regulations, Health claims: General requirements and Subpart E-Specific requirements for Health Claims. Washington: U.S. Government Printing Office; 2002. [Revised as of April 1].

15. Brasil. Ministério da Saúde (MS). Guia alimentar para a população brasileira: promovendo a alimentação saudável. $2^{\text {a }}$ ed. Brasília: MS; 2014.

16. Drewnowski A. The contribution of milk and milk products to micronutrient density and affordability of the U.S. diet. J Am Coll Nutr 2011; 30(5 Supl. 1):422S-428S.

Artigo apresentado em 05/12/2017

Aprovado em 19/07/2018

Versão final apresentada em 21/07/2018 
